\title{
An exact solution on the ferromagnetic Face-Cubic spin model on a Bethe lattice.
}

\author{
V. R. Ohanyan ${ }^{1,2}$, L. N. Ananikyan ${ }^{1}$, N. S. Ananikian ${ }^{1}$ \\ ${ }^{1}$ Department of Theoretical Physics, Yerevan Physics Institute, \\ Alikhanian Brothers 2, 375036 Yerevan, Armenia \\ ${ }^{2}$ Chair of Theoretical Physics, Yerevan State University, \\ Al. Manoogian 1, 375049, Yerevan, Armenia
}

September 2, 2018

\begin{abstract}
The lattice spin model with $Q$-component discrete spin variables restricted to have orientations orthogonal to the faces of $Q$-dimensional hypercube is considered on the Bethe lattice, the recursive graph which contains no cycles. The partition function of the model with dipole-dipole and quadrupole-quadrupole interaction for arbitrary planar graph is presented in terms of double graph expansions. The latter is calculated exactly in case of trees. The system of two recurrent relations which allows to calculate all thermodynamic characteristics of the model is obtained. The correspondence between thermodynamic phases and different types of fixed points of the RR is established. Using the technique of simple iterations the plots of the zero field magnetization and quadrupolar moment are obtained. Analyzing the regions of stability of different types of fixed points of the system of recurrent relations the phase diagrams of the model are plotted. For $Q \leq 2$ the phase diagram of the model is found to have three tricritical points, whereas for $Q>2$ there are one triple and one tricritical points.
\end{abstract}

PACS number(s): 05.50.+q, 05.70.Fh 


\section{Introduction}

Cubic symmetry plays a significant role in many types of phase transitions and critical phenomena. Still in 70-s various models possessing cubic symmetry have been introduced to determine the nature of the displacive phase transitions in perovskites [1, 2]. Magnetic phenomena in cubic crystals are also affected by the lattice structure. For instance, in the crystalline solids with cubic-symmetric lattices (Fe, $\mathrm{Ni}$, etc.) it leads to the modification of the magnetic exchange interaction giving rise to additional contributions to the conventional $\mathrm{O}(\mathrm{N})$-symmetric Heisenberg Hamiltonian. The simplest contribution of the underlying cubic symmetry is the single-ion anisotropy of the form $\sum_{i} \mathbf{s}_{\mathbf{i}}^{\mathbf{4}}$. During the last three decades different aspects of this issue have been the subject of various investigations.

In the framework of the field-theoretical approach to critical phenomena modelling of effects of cubic symmetry is usually performed in terms of continuous-spin Landau-Ginzburg effective Hamiltonian with cubic anisotropy, i.e. the $\phi^{4}$ - theory with an additional cubic term which breaks explicitly the $\mathrm{O}(\mathrm{N})$ invariance to a residual discrete cubic symmetry [3] - [7]:

$$
-\beta \mathcal{H}=\int d^{D} x\left\{\frac{1}{2}\left(\partial_{\mu} \phi\right)^{2}+\frac{1}{2} a \phi^{2}+\frac{1}{4} b \phi^{4}+v \sum_{\alpha=1}^{N}\left(\phi_{\alpha}\right)^{4}\right\},
$$

where $\phi(x)=\left(\phi_{1}, \phi_{2}, \ldots, \phi_{N}\right)$ is a continuous $\mathrm{N}$-component local vector order parameter, $\phi^{4}=\left(\phi^{2}\right)^{2}$ and $\beta=1 / k T$ is inverse temperature. The spin orientations, orthogonal to faces of an $N$-dimensional hypercube will be favorable for $v<0$, whereas positive values of $v$ favor the orientations toward the corners.

This model received numerous important applications, among which are such as the oxygen ordering in $\mathrm{YBaCu}_{3} \mathrm{O}_{6+x}$ [8], one of the most studied high- $\mathrm{T}_{c}$ superconductor; the buckling instability of confined colloid crystal layer [9]; the micellar binary solution of water and amphiphile [10] etc. The two-dimensional case has been recently intensively studied within the framework of renormalization group technique in the space of fixed dimensionality up to fiveloop approximation [5]-[7]. The model was found to have four fixed points: the Gaussian one, the Ising one with $\mathrm{N}$ decoupled components, the $\mathrm{O}(\mathrm{N})$-symmetric and the cubic fixed point. Analogous calculations were made for $D=3$, as well revealing some peculiar type of cubic fixed point for $N>2$ corresponding to specific anisotropic mode of the critical behavior [11]-[15]. The surface critical behavior of the model was also studied [16]. It has been recently shown that the behavior of the spherical many-spin magnetic nanoparticle with surface anisotropy may be modelled by an effective single macro-spin with cubic anisotropy terms in the effective energy [17].

Another class of lattice cubic-symmetric spin models was introduced by Kim, Levy and Uffer in Ref. [18] to explain the tricritical-like behavior of cubic rare-earth compounds, particularly holmium antimonide, HoSb [18]-[23]. Projecting the pair exchange interaction onto the sixfold degenerated ground-state manifold of $\mathrm{Ho}^{3+}$ ion they arrived at the following effective 
Hamiltonian:

$$
-\beta \mathcal{H}=\sum_{\langle i, j\rangle} J_{i j} \mathbf{S}_{i} \mathbf{S}_{j},
$$

where the classical spin variables $\mathbf{S}_{i}$ are the unit vectors restricted to have orientations, orthogonal to the faces of the cube and the sum is going over all the pairs of nearest-neighbor sites. Generalization for $Q$ component spin is obvious: each spin can assume $2 Q$ orientations:

$$
\mathbf{S}_{i} \in\{( \pm 1,0, \ldots, 0),(0, \pm 1, \ldots, 0) \ldots(0, \ldots, 0, \pm 1)\} .
$$

This model is known as the Face-cubic model. It is connected to the continuous cubic model of Eq. (1) via the limit of strong anisotropy $(|v| \gg|b|)$. In a similar way one can also consider quadrupolar pair interaction terms and obtain the following Hamiltonian provided all interactions are homogenous [20,21]:

$$
-\beta \mathcal{H}_{F C}=J \sum_{\langle i, j\rangle}\left(\mathbf{S}_{i} \mathbf{S}_{j}\right)+K \sum_{\langle i, j\rangle}\left(\mathbf{S}_{i} \mathbf{S}_{j}\right)^{2} .
$$

It is easy to see that Hamiltonian (4) can be represented in terms of two sets of discrete variables: a Potts-like determining which component of $\mathbf{S}_{i}$ is non-zero and an Ising-like, corresponding to the sign of the component. Indeed,

$$
\mathbf{S}_{i} \mathbf{S}_{j}=\sigma_{i} \sigma_{j} \delta_{\alpha_{i} \alpha_{j}},
$$

where $\sigma_{i}= \pm 1$ and $\alpha_{i}=1,2, \ldots Q$. Therefore we have

$$
-\beta \mathcal{H}_{F C}=J \sum_{\langle i, j\rangle} \sigma_{i} \sigma_{j} \delta_{\alpha_{i}, \alpha_{j}}+K \sum_{\langle i, j\rangle} \delta_{\alpha_{i}, \alpha_{j}} .
$$

Formally we can enlarge the Hamiltonian (4) up to the interaction terms of higher power of the $\left(\mathbf{S}_{i} \mathbf{S}_{j}\right)$ :

$$
-\beta \mathcal{H}_{F C}^{(L)}=\sum_{\langle i, j\rangle} \sum_{n=0}^{L} J_{n}\left(\mathbf{S}_{i} \mathbf{S}_{j}\right)^{n} .
$$

For arbitrary finite $L$ this expression leads to the same Hamiltonian that of Eq. (6) with $J=\sum_{k=0}^{L} J_{2 k+1}$ and $K=\sum_{k=0}^{L} J_{2 k}$. At $K=0$ the Hamiltonian (7) reduced to the $2 Q-$ state Potts model; $J=0$ corresponds to two decoupled $Q$ - state Potts model, and at $Q=2$ one obtains the Ashkin-Teller model. Variational renormalization-group study of the pure and diluted $Q$ - component face-cubic model in two dimensions has revealed existence of four competing possible types of critical behavior corresponding to $Q$ - state Potts model, $2 Q-$ state Potts model, Ising model and special "cubic" fixed point [22, 23]. It was also found that at $Q<Q_{c}=2$ the transitions are continuous and critical behavior of the discrete face-cubic 
model belongs to the $\mathrm{O}(\mathrm{N})$-model universality class. For $Q=2$ the Ashkin-Teller-like behavior occurs and for $Q>2$ transitions are of first order. There are also early results obtained within the mean-field (MF) theory [18] and using the Bethte-Peirels (BP) approximations and hightemperature series [19] for the case when only dipolar pair interactions are included $(K=0)$. In the $\mathrm{BP}$ approximation the critical value of spin component $Q_{c}$, above which transitions are of the first order was found to be given by

$$
Q_{c}=1+\frac{2}{3} q\left[\left(1+\frac{6}{q}\right)^{1 / 2}-1\right],
$$

where $q$ is the coordination number of the lattice. The limit $q \rightarrow \infty$ corresponds to the MF solution and gives $Q_{c}=3$. In Ref. [19] the high-temperature series for $Q$ - component facecubic model on three dimensional fcc lattice were constructed up to 5-th order, from which authors obtained $Q_{c}=2.35 \pm 0.2$, whereas Eq. (8) gives us $Q_{c} \simeq 2.8$ Moreover, although the MF solution of cubic model predicted the tricritical like behavior [18], the BP approximation showed that it is not the case [19]. Only inclusion of single-ion-anisotropy terms, quadrupolar pair interactions and crystal fields may drive the system tricritical [20].

The paper is organized as follows. In Section II we give the introduction to recursive lattice constructions and their role in statistical mechanics. Then we discuss the peculiarities of the Cayley tree, Bethe lattice and connections between these two objects. Section III is devoted to the graph expansions for the partition function of the model under consideration defined on the arbitrary planar graph. We presented the partition function in terms of double power series which in case of the Cayley tree are summed up exactly, giving the same expression as in case of one-dimensional linear chain. We also discuss arguments of Eggarter concerning the mechanisms of phase transitions on the Cayley tree and Bethe lattice. In the next Section IV we derive the system of recurrent relations for the $F C_{Q}$-model on the Bethe lattice reformulating the statistical-mechanical problem in terms of the theory of dynamical systems [25]. We introduce the order parameters and give their expressions in terms of recursive scheme. In Section $\mathrm{V}$ we identify different types of the fixed points of the system of recurrent relation with different physical phases and present the plots of order parameters temperature dependencies. The phase diagrams for different values of spin component number $Q$ are also presented. Brief summary is given in Section VI.

\section{Recursive methods, Cayley tree and Bethe lattice}

Among the vast variety of statistical mechanics lattice models with strong local interactions only very limited amount allows exact solutions. These exact solutions are known only for low-dimensional systems, more precisely for $d=1$ and $d=2$; most of exact solutions for twodimensional systems are known only in the absence of the external field and/or for the special choice of model parameters [24]. Well known conventional approximate methods like MF theory and BP approximation in general can provide only more or less qualitatively satisfactory picture 


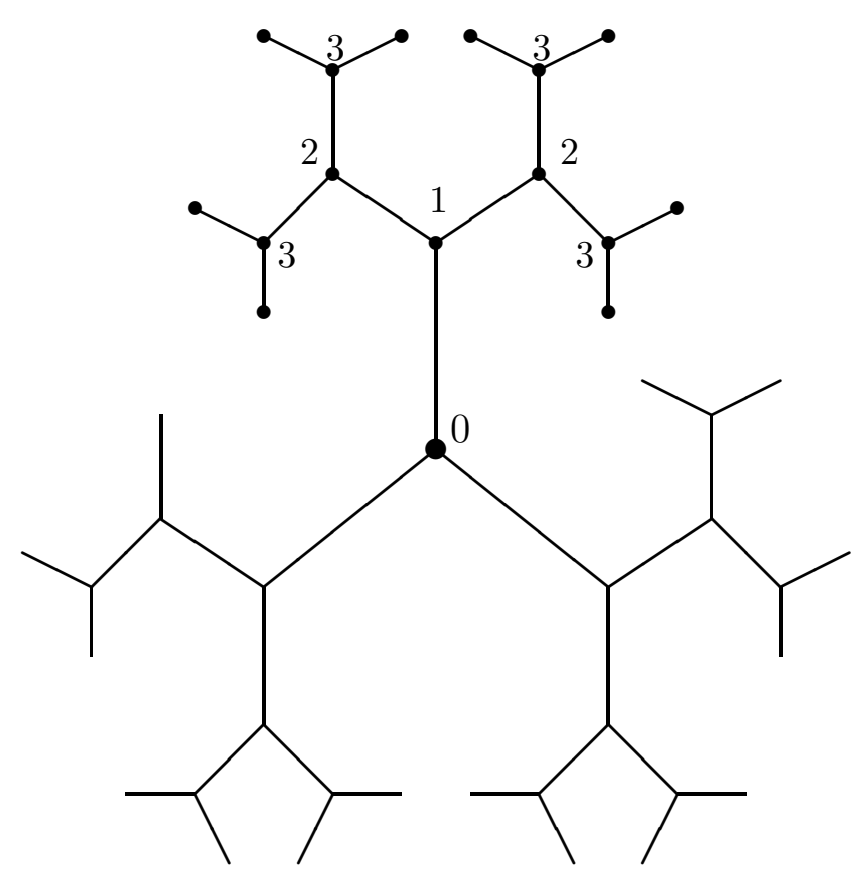

Figure 1: The Cayley tree with coordination number $q=3$ and 3 shells.

and in some cases they just fail. That is why the quest for the alternative approaches, which can provide more reliable results for the thermodynamics of lattice models is very important.

The recursive lattices are of twofold interest. On the one hand, the models, defined on a recursive lattices can be considered as an independent original area of research in which the powerful methods of dynamical systems theory and fractal geometry are successfully exploited for determining the thermodynamical properties of the statistical mechanics models. On the other hand, recursive lattices provide a specific kind of approximate treatment of many-particle systems physics. In the heart of these approximations not the simplification of the character or/and strength of the interaction between the system elements, but the modification of the topology of the underlying lattice lies. This modification in most cases consist in the replacement of the regular periodic "physical" lattice by a recursive one, constructed by the certain algorithm and possessing the self-similarity, maintaining all interaction unchanged. For the large class of lattice models with commuting variables ("classical models") this approach leads to the exact solution of the statical mechanics problem in terms of the theory of dynamical systems or, more precisely, in terms of discrete maps. The solution formally is rather similar to that which corresponds to conventional BP approximation but, in contrast to the latter, it is the exact solution for the lattice endowed with the recursive structure provided the boundary sites are properly taken into consideration. It was argued that in some cases, particularly in the 
models where multi-site interactions are presented [31]-[33] the recursive lattice approximation gives more reliable results than conventional $\mathrm{BP}$ [34].

The simplest example of recursive lattices is a Cayley tree, the self-similar graph, which contains no cycles. In order to construct a Cayley tree one should start with a central site $O$. At the first step this site should be connected by links with $q$ others, which constitute the first shell or the first generation of the Cayley tree. The second shell of the Cayley tree is constructed by repeating this procedure for all sites of the first shell: each of the $q$ sites belonging to the first shell connects to the $q-1$ new sites. Thus, the second shell contains $N_{2}=q(q-1)$ sites. Accomplishing this construction for $\mathrm{n}$ steps one arrives at the recursive connected graph, which contains no cycles and is called the Cayley tree with coordination number $q$ and $n$ generations (shells) (Fig. 1). As the number of sites in $k$-th shell is $q(q-1)^{k-1}$ the total number of sites then

$$
N_{n}=1+\sum_{k=1}^{n} q(q-1)^{k-1}=\frac{q(q-1)^{n}-2}{q-2} .
$$

Thus, each site of the Cayley tree has coordination number equal to $q$ except the sites on the last shell which have only one neighbor. The peculiar property of the Caylet tree is the large amount of boundary sites which even in the thermodynamical limit $n \rightarrow \infty$ comprise a finite fraction of the total number of sites

$$
\lim _{n \rightarrow \infty} \frac{q(q-1)^{n-1}}{N_{n}}=\lim _{n \rightarrow \infty} \frac{(q-2) q(q-1)^{n-1}}{q(q-1)^{n}-2}=\frac{q-2}{q-1} .
$$

This feature causes anomalous properties of the statistical mechanics systems on the full Cayley tree. For instance, in Ising model there is no zero field magnetization, whereas the derivations of free energy with respect to the external field exhibit singular behavior [26] - [29].

The Bethe lattice is an object intimately linked to the Cayley tree but, in contrast to the latter, it is devoid of the complications which arise from the boundary sites. When one deals with the Bethe lattice the general structure and topology of the Cayley tree are preserved whereas only contributions from the bulk sites lying deep inside the Cayley tree are taken into consideration. In this regard the Bethe lattice is the interior of the Cayley tree [24, 30]. Dealing with the Bethe lattice we suppose the underlying Cayley tree to be large enough to achieve thermodynamical limit and consider only the sites situated far away from a boundary. One can regard these sites as a uniform lattice with coordination number $q$.

An undeniable advantage of the Bethe lattice is the possibility of exact solutions for many types of statistical mechanics problems. A large amount of problems of the theory of magnetism, macromolecule physics, lattice gauge theory, self-organized criticality, dynamical mean-field theory (DMFT) and general questions of statistical mechanics like zeroes of partition functions have been successfully considered on Bethe or Bethe-like lattices, revealing many interesting exact results and deep connections between the theory of dynamical systems and statistical mechanics [26] - [49]. 


\section{$3 \quad$ Face-cubic model on planar graphs}

It is possible to calculate exactly the partition function of the $F C_{Q}$-model on Cayley tree in case of the absence of external field. Let us consider the partition function of the model without external field on arbitrary planar graph $G$

$$
\mathcal{Z}_{G}=\sum_{\{\sigma\}} \sum_{\{\alpha\}} \prod_{\langle i, j\rangle \in G} \exp \left\{\left(J \sigma_{i} \sigma_{j}+K\right) \delta_{\alpha_{i}, \alpha_{j}}\right\}
$$

As in the case of Potts model [50] one can represent it in the following way:

$$
\mathcal{Z}_{G}=\sum_{\{\sigma\}} \sum_{\{\alpha\}} \prod_{\langle i, j\rangle \in G}\left(1+U_{\sigma_{i} \sigma_{j}} \delta_{\alpha_{i}, \alpha_{j}}\right)
$$

where $U_{\sigma_{i} \sigma_{j}}=e^{J \sigma_{i} \sigma_{j}+K}-1$. Then summing out over the all $\alpha_{i}$ we obtain that

$$
\mathcal{Z}_{G}=\sum_{\{\sigma\}} \sum_{G^{\prime} \subseteq G} Q^{R_{0}\left(G^{\prime}\right)} \prod_{\langle i, j\rangle \in G^{\prime}} U_{\sigma_{i} \sigma_{j}}
$$

where the second sum is going over all spanning graphs $G^{\prime}$ of the underlying planar graph $G$ and $R_{0}\left(G^{\prime}\right)$ is the 0-th Betti's number of $G^{\prime}$ which coincides with the number of connected components of $G^{\prime}$ and the product is over all links $\langle i, j\rangle$ belonging to $G^{\prime}$. Using the identity

$\sigma_{i} \sigma_{j}=2 \delta_{\sigma_{i} \sigma_{j}}-1$ one can represent Eq. (13) in terms of double power series associated with the underlying lattice provided latter is a planar graph:

$$
\begin{aligned}
\mathcal{Z}_{G} & =\sum_{G^{\prime} \subseteq G} Q^{R_{0}\left(G^{\prime}\right)} u^{e\left(G^{\prime}\right)} \sum_{\{\sigma\}} \prod_{\langle i, j\rangle \in G^{\prime}}\left(1+v \delta_{\sigma_{i} \sigma_{j}}\right), \\
u & =e^{K-J}-1 \\
v & =\frac{e^{2 J}-1}{1-e^{J-K}}
\end{aligned}
$$

where $e\left(G^{\prime}\right)$ is the edges number in the graph $G^{\prime}$ and all $\sigma_{i}$ assume values 0 or 1 . So, for each spanning subgraph $G^{\prime}$ of planar graph $G$ we obtain a partition function of 2-state Potts model:

$$
\begin{aligned}
\mathcal{Z}_{G} & =\sum_{G^{\prime} \subseteq G} Q^{R_{0}\left(G^{\prime}\right)} u^{e\left(G^{\prime}\right)} \mathcal{Z}_{G^{\prime}}^{\text {Potts }}(v) \\
& =\sum_{G^{\prime} \subseteq G} Q^{R_{0}\left(G^{\prime}\right)} u^{e\left(G^{\prime}\right)} \sum_{G^{\prime \prime} \subseteq G^{\prime}} 2^{R_{0}\left(G^{\prime \prime}\right)} v^{e\left(G^{\prime \prime}\right)},
\end{aligned}
$$

where the second sum is going over all subgraph of $G^{\prime}$ Thus, we have succeeded in representing the partition function of the $F C_{Q}$ - model given by Hamiltonian (6) in terms of double power series associated with the underlying lattice provided the latter is a planar graph. As in case of ordinary spin Potts model one can make $1 / Q$-expansions for $F C_{Q}$-model with the aid of Eq. 
(15). For instance, for the two-dimensional square lattice one obtains

$$
\begin{aligned}
\mathcal{Z} & =(2 Q)^{N}\left\{1+2 N \frac{u}{Q}\left(\frac{v}{2}+1\right)+N(2 N-1)\left(\frac{u}{Q}\right)^{2}\left(\frac{v}{2}+1\right)^{2}+C_{2 N}^{3}\left(\frac{u}{Q}\right)^{3}\left(\frac{v}{2}+1\right)^{3}\right. \\
& +\left(C_{2 N}^{4}-N\right)\left(\frac{u}{Q}\right)^{4}\left(\frac{v}{2}+1\right)^{4}+N \frac{u^{4}}{Q^{3}}\left(\left(\frac{v}{2}+1\right)^{4}+\left(\frac{v}{2}\right)^{4}\right)+\left(C_{2 N}^{5}-N(2 N-4)\right)\left(\frac{u}{Q}\right)^{5}\left(\frac{v}{2}+1\right)^{5} \\
& +N(2 N-4) \frac{u^{5}}{Q^{4}}\left(\left(\frac{v}{2}+1\right)^{5}+\left(\frac{v}{2}\right)^{4}+\left(\frac{v}{2}\right)^{5}\right) \\
& \left.+2 N \frac{u^{7}}{Q^{5}}\left(\left(\frac{v}{2}+1\right)^{7}+2\left(\left(\frac{v}{2}\right)^{4}+\left(\frac{v}{2}\right)^{5}\right)+3\left(\frac{v}{2}\right)^{6}\right)+\ldots\right\} .
\end{aligned}
$$

Now we can use the well known relations between the topological invariants of graphs [51] to rewrite Eq. (15) in another way suitable for our further purposes. Applying the Euler theorem to planar graph $G$ we get

$$
R_{0}(G)-R_{1}(G)=n-e(G)
$$

where the first Betti's number $R_{1}(G)$ coincides with the number of independent cycles of $G$ and $n$ is the number of sites. Therefore

$$
\mathcal{Z}_{G}=(2 Q)^{n} \sum_{G^{\prime} \subseteq G} Q^{R_{1}\left(G^{\prime}\right)}\left(\frac{u}{Q}\right)^{e\left(G^{\prime}\right)} \sum_{G^{\prime \prime} \subseteq G^{\prime}} 2^{R_{1}\left(G^{\prime \prime}\right)}\left(\frac{v}{2}\right)^{e\left(G^{\prime \prime}\right)} .
$$

\subsection{The case of graphs without cycles: trees and forests}

Partition function for $F C_{Q}$-model in the form of Eq. (18) can easily be calculated in case of the so-called forests, the planar graphs, which contain no cycles. Connected part of forest is called tree. Let us suppose that we have a tree $T$ with $n$ sites and $L_{n}$ edges. Then Eq.(18) takes the form

$$
\begin{aligned}
\mathcal{Z}_{T} & =(2 Q)^{n} \sum_{G^{\prime} \subseteq T}\left(\frac{u}{Q}\right)^{e\left(G^{\prime}\right)} \sum_{G^{\prime \prime} \subseteq G^{\prime}}\left(\frac{v}{2}\right)^{e\left(G^{\prime \prime}\right)} \\
& =(2 Q)^{n} \sum_{k=0}^{L_{n}} C_{L_{n}}^{k}\left(\frac{u}{Q}\right)^{k} \sum_{l=0}^{k} C_{k}^{l}\left(\frac{v}{2}\right)^{l}
\end{aligned}
$$

where $C_{k}^{l}=\frac{k !}{l !(k-l) !}$ are the binomial coefficients and the sum is going over all subgraphs with given volume $k$. With the aid of Newton's binom we obtain

$$
\mathcal{Z}_{T}=(2 Q)^{n}\left(\frac{u}{Q}\left(\frac{v}{2}+1\right)+1\right)^{L_{n}} .
$$

If $T$ is the Cayley tree then $L_{n}=n-1$ and we will have

$$
\mathcal{Z}_{\text {Cayley }}=2 Q(u(v+2)+2 Q)^{n-1} .
$$


Thus, the free energy per site for $F C_{Q}$-model on Cayley tree is

$$
\begin{array}{r}
f=-k_{B} T \lim _{n \rightarrow \infty} \frac{\log \mathcal{Z}_{\text {Cayley }}}{n}=-k_{B} T \log (u(v+2)+2 Q) \\
=-k_{B} T \log 2-k_{B} T \log \left(e^{K} \cosh J+Q-1\right) .
\end{array}
$$

It is noteworthy that this result is in full agreement with that obtained by Aharony for one-dimensional model by transfer-matrix technique [21]. Indeed, a one-dimensional chain can be regarded as a "tree" in the sense mentioned above. Thus, the free energy of the $F C_{Q}$ model on a Cayley tree in a thermodynamic limit is continuous for all $T$. Formally it coincides with that for one-dimensional systems where the continuity is really the case. But, as is known, the BP approximations for the lattice model with an arbitrary number of component solves exactly the problem on a Cayley tree [40]. Therefore the model on a Cayley tree must possess a finite critical temperature as predicted by BP approximation [19]. The origin of this peculiar properties of the Cayley tree was revealed by Eggarter [26] on the example of Ising model. He argued that the equivalence of all sites in the thermodynamic limit, which is one of the key points of BP approximation, breaks down for the sites of the Cayley tree which are situated close to the surface. As appears from Eq. (10) these sites comprise rather large fraction of the sites of the Cayley tree and, thus, they determine the behavior of all thermodynamic quantities to a great extent.

However, even though no phase transitions in the thermodynamic limit occur at the value of critical temperature $T_{c}$ predicted by the BP method the value of the order parameter (magnetization etc.) in the interior of the lattice, i. e. for the region far from the surface, which is generally called "Bethe lattice", will undergo jumps from zero to its BP value when the temperature is below $T_{c}$. In order to illustrate it we will use the technique of dynamical system theory, which becomes a powerful tool for investigating various physical problems on recursive lattices.

\section{Recursive method for Face Cubic model on Bethe lat- tice.}

Many statistical systems defined on the recursive lattices are famous for the possibility of exact solution in terms of dynamical system theory. In the heart of these exact solutions the self-similarity of recursive lattices lies. For instance, if we cut apart the Cayley tree at the central site it will give q identical branches each of which is the same Cayley tree with the number of generation decreased by 1 . Using this fact one can establish the connection between the partition function of a model defined on the Cayley tree containing $\mathrm{n}$ shells with the partition function of the same model defined on the Cayley tree containing n-1 shells. Therefore, the thermodynamical problem is reformulated in terms of discrete maps, given by recurrent relations. Let us introduce a symmetry breaking field into Hamiltonian (6). The 
field can be considered as a uniform magnetic field pointing along the first coordinate axis $\mathbf{H}=(H, 0, \ldots 0)$. The corresponding interaction Hamiltonian is of the conventional Zeeman type:

$$
\mathcal{H}_{Z}=-\mathbf{H} \sum_{i} \mathbf{S}_{i}
$$

which leads to the following form of the $F C_{Q^{-}}$model Hamiltonian in the field:

$$
-\beta \mathcal{H}_{F C}=J \sum_{\langle i, j\rangle} \sigma_{i} \sigma_{j} \delta_{\alpha_{i}, \alpha_{j}}+K \sum_{\langle i, j\rangle} \delta_{\alpha_{i}, \alpha_{j}}+h \sum_{i} \sigma_{i} \delta_{\alpha_{i}, 1}
$$

where $h=\beta H$. Hereafter we pass from the Cayley tree to the Bethe lattice having in mind one of the ways mentioned in Section II. Thus, we should no longer care about boundary sites and boundary conditions. According to that one can represent the partition function of the $F C_{Q}$-model on the Bethe lattice in the following form:

$$
Z=\sum_{\left(\sigma_{0}, \alpha_{0}\right)} e^{h \sigma_{0} \delta_{\alpha_{0}, 1}}\left[g_{n}\left(\sigma_{0}, \alpha_{0}\right)\right]^{q},
$$

where $\sigma_{0}$ and $\alpha_{0}$ are the variables of the spin in the central site and $g_{n}\left(\sigma_{0}, \alpha_{0}\right)$ refers to a partition function of individual branch:

$$
g_{n}\left(\sigma_{0}, \alpha_{0}\right)=\sum_{\sigma \neq \sigma_{0}} \sum_{\alpha \neq \alpha_{0}} \exp \left(\left(J \sigma_{0} \sigma_{1}+K\right) \delta_{\alpha_{0}, \alpha_{1}}+\sum_{\langle i, j\rangle}\left(J \sigma_{i} \sigma_{j}+K\right) \delta_{\alpha_{i}, \alpha_{j}}+\sum_{i} \sigma_{i} \delta_{\alpha_{i}, 1}\right) .
$$

Each branch, in its turn, can be cut at the site which was previously connected to the central one. This will give us q-1 identical branches being the Bethe lattices with n- 1 generations. Thus, the connection between $g_{n}$ and $g_{n-1}$ is

$$
g_{n}\left(\sigma_{0}, \alpha_{0}\right)=\sum_{\left(\sigma_{1}, \alpha_{1}\right)} \exp \left(\left(J \sigma_{0} \sigma_{1}+K\right) \delta_{\alpha_{0}, \alpha_{1}}+h \sigma_{1} \delta_{\alpha_{1}, 1}\right)\left[g_{n-1}\left(\sigma_{1}, \alpha_{1}\right)\right]^{q-1},
$$

Here we obtain the system of $2 Q$ recursion relations but, in fact, only three of them are independent as all $g_{n}(\sigma, \alpha)$ for $\alpha \neq 1$ are identical. So, from (27) we have

$$
\begin{aligned}
& g_{n}(+, 1)=e^{J+K+h}\left[g_{n-1}(+, 1)\right]^{q-1}+e^{-J+K-h}\left[g_{n-1}(-, 1)\right]^{q-1}+2(Q-1)\left[g_{n-1}( \pm, *)\right]^{q-1}, \\
& g_{n}(-, 1)=e^{-J+K+h}\left[g_{n-1}(+, 1)\right]^{q-1}+e^{J+K-h}\left[g_{n-1}(-, 1)\right]^{q-1}+2(Q-1)\left[g_{n-1}( \pm, *)\right]^{q-1}, \\
& g_{n}( \pm, *)=e^{h}\left[g_{n-1}(+, 1)\right]^{q-1}+e^{-h}\left[g_{n-1}(-, 1)\right]^{q-1}+\left(e^{J+K}+e^{-J+K}+2(Q-2)\right)\left[g_{n-1}( \pm, *)\right]^{q-1},
\end{aligned}
$$

where $g_{n}( \pm, *)$ stand for any 2Q-2 partition functions corresponding to the individual branch with $\vec{S}_{1}$ whose direction is non collinear with the first coordinate axes. Introducing the variables

$$
\begin{gathered}
x_{n}=g_{n}(+, 1) / g_{n}( \pm, *), \\
y_{n}=g_{n}(-, 1) / g_{n}( \pm, *),
\end{gathered}
$$


we obtain the system of two recurrent relations

$$
\begin{gathered}
x_{n}=f_{1}\left(x_{n-1}, y_{n-1}\right), \\
y_{n}=f_{2}\left(x_{n-1}, y_{n-1}\right),
\end{gathered}
$$

with

$$
\begin{aligned}
& f_{1}(x, y)=\frac{P_{1}(x, y)}{R(x, y)}=\frac{a \mu x^{q-1}+b \mu^{-1} y^{q-1}+2(Q-1)}{\mu x^{q-1}+\mu^{-1} y^{q-1}+a+b+2(Q-2)}, \\
& f_{2}(x, y)=\frac{P_{2}(x, y)}{R(x, y)}=\frac{b \mu x^{q-1}+a \mu^{-1} y^{q-1}+2(Q-1)}{\mu x^{q-1}+\mu^{-1} y^{q-1}+a+b+2(Q-2)},
\end{aligned}
$$

where the following notations are adopted

$$
\begin{aligned}
a & =\exp (J+K) \\
b & =\exp (-J+K) \\
\mu & =\exp (h)
\end{aligned}
$$

In this approach statistical averages of all physical quantities can be expressed in terms of $x$ and $y$ variables defined by Eq. (30). For instance, when the total number of spins is $n$ the magnetization along the first coordination axis which is the thermal average of the following form:

$$
m=\frac{1}{n} \sum_{i=1}^{n}\left\langle S_{i}^{(1)}\right\rangle=\frac{1}{n} \sum_{i=1}^{n}\left\langle\sigma_{i} \delta\left(\alpha_{i}, 1\right)\right\rangle
$$

in terms of $x$ and $y$ are expressed as

$$
m=\frac{\mu x^{q}-\mu^{-1} y^{q}}{\mu x^{q}+\mu^{-1} y^{q}+2(Q-1)},
$$

provided all sites of the lattice are equivalent. It is easy to see that this quantity plays the role of an order parameter of Ising universality class. Another order parameter belonging to the $Q$-state Potts universality class is

$$
p=\frac{1}{n} \sum_{i=1}^{n}\left\langle\delta\left(\alpha_{i}, 1\right)\right\rangle,
$$

which also can be regarded as a "quadrupolar" moment $\left\langle S_{i}^{(1)^{2}}\right\rangle$. Thus

$$
p=\frac{\mu x^{q}+\mu^{-1} y^{q}}{\mu x^{q}+\mu^{-1} y^{q}+2(Q-1)} .
$$


The order parameters $m$ and $p$ define the three possible phases of the $F C_{Q}$ model in case of the ferromagnetic couplings, i.e. $J>0, K>0$, provided external magnetic field is vanished:

(a) disordered (paramagnetic) phase: $m=0, p=1 / Q$

(b) ferromagneticaly ordered phase: $m \neq 0, p \neq 1 / Q$

(c) partially ordered (quadrupolar) phase: $m=0, p \neq 1 / Q$

\section{Investigations of phase transitions in terms of dynam- ical systems theory}

In order to obtain physical results one must implement an iterative procedure for the RR (31). Namely, starting from the random initial conditions $\left(x_{0}, y_{0}\right)$ one uses the simple iterations scheme and examines the behavior of physical quantities after a large number of iterations [35]. In a simplest case the iterative sequence $\left\{x_{n}, y_{n}\right\}$ converges to a fixed point $\left(x^{*}, y^{*}\right)$, which is defined by

$$
\left\{\begin{array}{l}
x^{*}=f_{1}\left(x^{*}, y^{*}\right) \\
y^{*}=f_{2}\left(x^{*}, y^{*}\right)
\end{array}\right.
$$

This situation is inherent to the ferromagnetic case when both $J$ and $K$ are positive. As can easily be seen from Eqs. (33) - (36), when the magnetization and quadrupolar moment of the system take values $m$ and $p$ respectively, then the corresponding fixed point of the RR (31) is the following, provided $h=0$ :

$$
\left\{\begin{array}{l}
x^{*}=\left(\frac{(Q-1)(p+m)}{1-p}\right)^{1 / q} \\
y^{*}=\left(\frac{(Q-1)(p-m)}{1-p}\right)^{1 / q}
\end{array}\right.
$$

Thus, according to the properties of different phases of the ferromagnetic $F C_{Q}$ model one can establish the connection between them and the classification of the possible types of fixed points:

(a) disordered (paramagnetic) phase: $x^{*}=y^{*}=1$

(b) ferromagneticaly ordered phase: $x^{*} \neq y^{*} \neq 1$

(c) partially ordered (quadrupolar) phase: $x^{*}=y^{*} \neq 1$

The condition $x^{*}=y^{*}$ leads to $g_{n}(+, 1)=g_{n}(-, 1)$, which means that the probability of spin "up" is equal to the probability of spin "down" which is really the case in paramagnetic phase. 
(a)

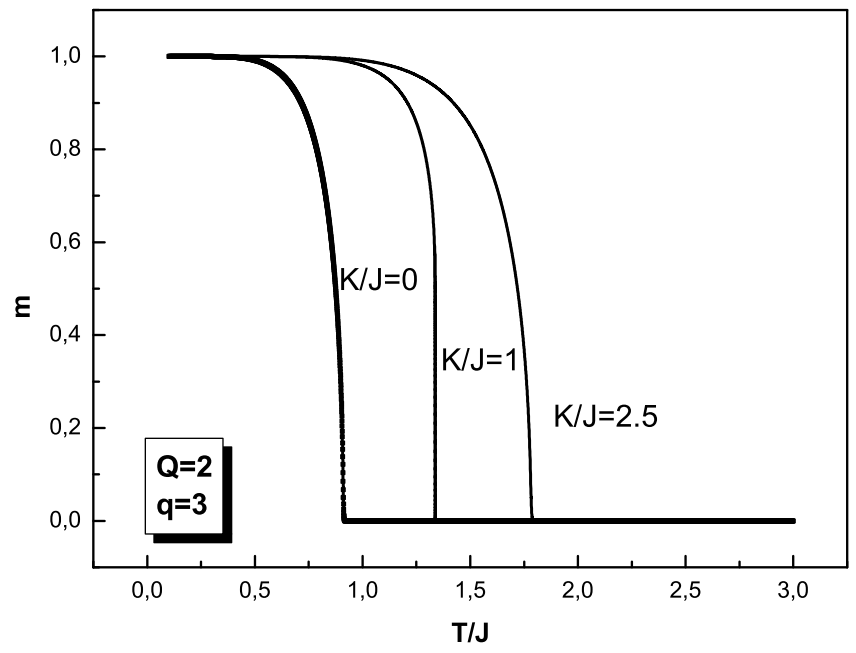

(b)

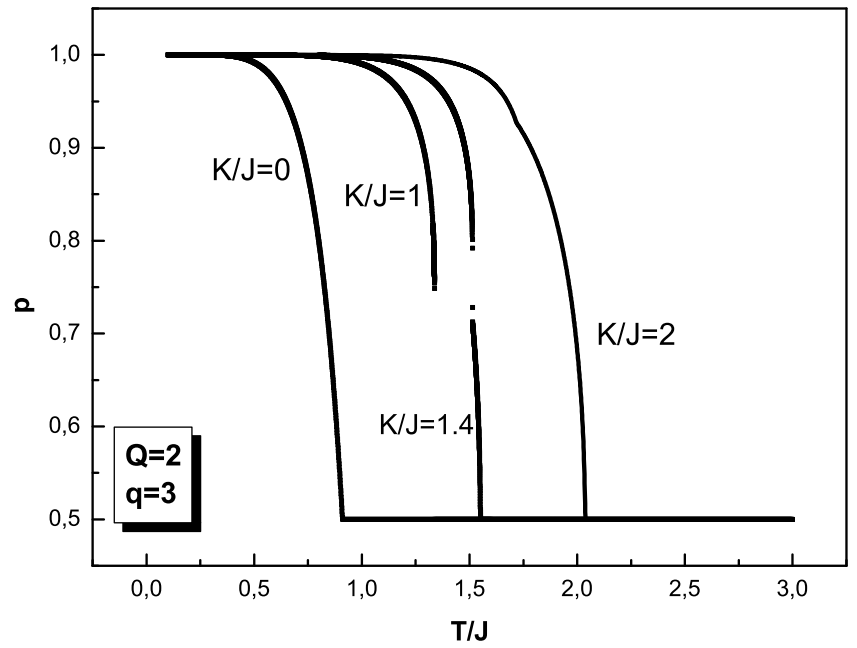

Figure 2: The temperature behavior of the order parameters for $Q=2$ and $q=3$. The transitions from disordered to ferromagnetic phase is of second order for all range of $K$.(a) Zero field magnetization for $K / J=0 ; 1$ and 2.5; (b) Quadrupolar moment in zero field for $K / J=0 ; 1 ; 1.4$ and 2 . For $K / J=1$ one can see the first order transition between disordered and ferromagnetic phases, whereas at $K / J=1.4$ one can see two subsequent phase transitions, the continuous transition from disordered to quadrupolar phase and very close to that the first order transition to the ferromagnetic phase. With the further increase of $K / J$ the second transition becomes of the second order.

Applying the simple iterative scheme to Eqs. (30), (34) and (36) one can obtain the plots of magnetization processes ( $m$ vs. $H$ at fixed values of $T$ ) as well as the zero field magnetization and quadrupolar moment of the system.

In Fig. 2 one can see the temperature dependencies of the order parameters of the $F C_{Q^{-}}$ model on Bethe lattice with coordination number $q=3$ and $Q=2$. As is obviously seen from Fig. 2(a) in this case the zero field magnetization is always continuous, whereas the behavior of $p$ is quite different. Depending on the value of ratio $K / J$ the $p(T)$ curve could be continuous or can include one first order transition point. In Fig. 2(b) one can see that at small values of $K / J$ only the one phase transition occurs from disordered phase to ferromagnetic phase omitting the partially ordered phase. This transition is of the second order until $K / J$ reaches some intermediate value above which one can see a discontinuity in the order parameter $p$ at critical temperature corresponding to the transition between disordered and ferromagnetic phases. At large values of $K / J$ the system undergoes successively two phase transitions at temperatures $T_{q}(K)$ and $T_{f}(K)$, the larger one corresponds to the transition between disordered and quandrupolar phases, the lower one - to the transitions from quandrupolar to ferromagnetic. Within this region of the values of $K$ one can see the second order transition from disordered 


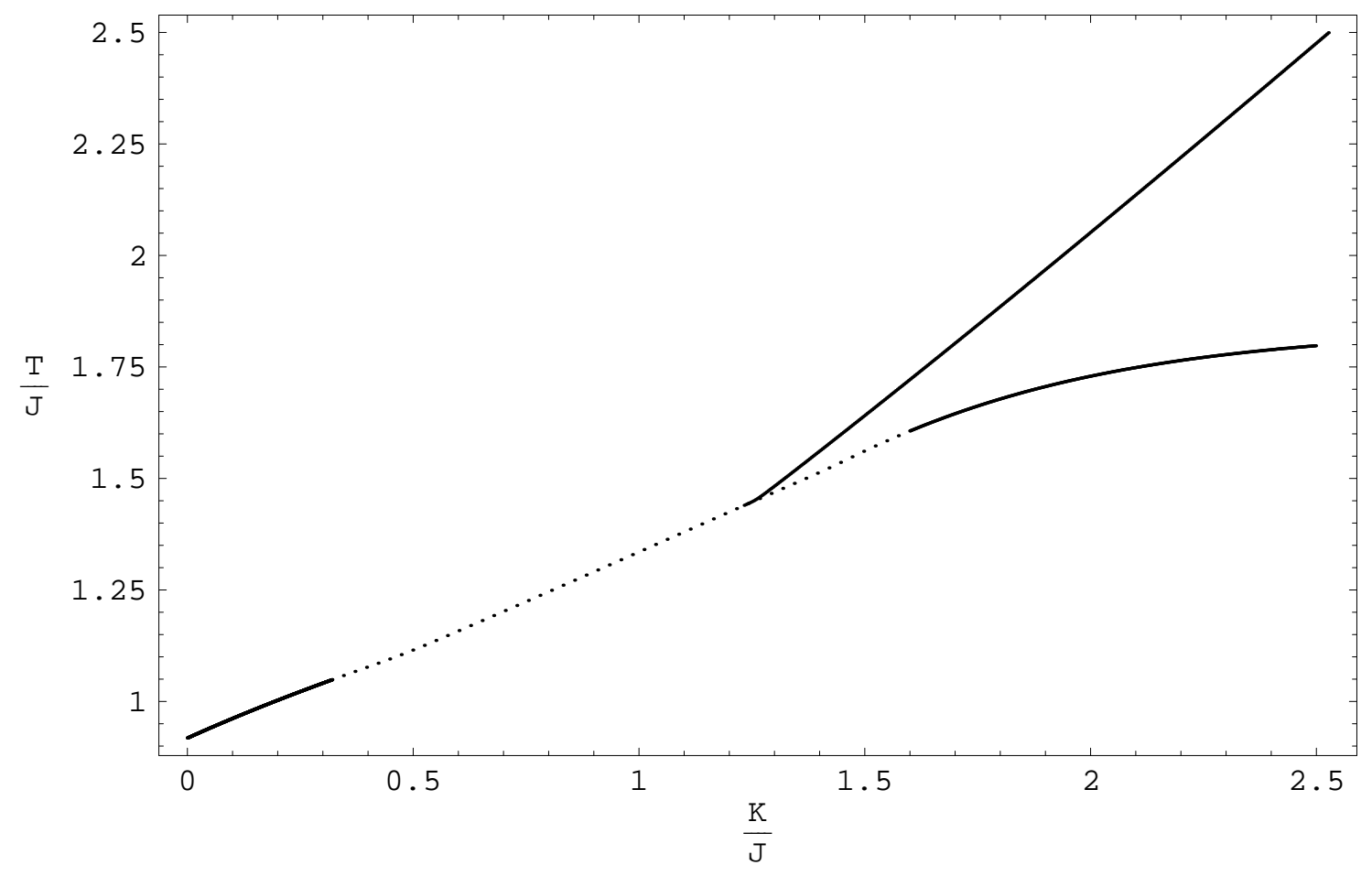

Figure 3: The phase diagram of the model for $Q=2$ and $q=3$. Solid line corresponds to the second order transitions, dotted - to the first order transitions. For small values of the ratio $K / J$ one can see only single phase transition line between disordered hight-temperature phase and completely ordered ferromagnetic phase, whereas for $K / J \geq 1.233$ there is the partially ordered "quadrupolar" phase between them. The tricritical points are $K / J \approx 0.32 ; 1.233$ and 1.6.

phase into "quadrupolar" phase and the further first order transition to the ferromagnetic phase. However, beginning with some value of $K$ the transition between quadrupolar and ferromagnetic phases becomes continuous.

In order to complete the picture of the phase structure of the model under consideration at $Q=2$ we plot a phase diagram by separating the regions of the fixed points of different kinds of the RR from Eq. (31) in the $(K / J, T / J)$-plane. One can see in Fig. 3 that in case of $Q=2$, $q=3$ at $K=0$ the phase transition from disordered to ferromagnetic phase is of the second order. This feature maintains up to $K / J \approx 0.32$, where one can notice the tricritical point separating the region of continuous transitions from the region of the first order transitions. The line of the second order phase transitions between disordered and quadrupolar phases merges the line of transitions between disordered and ferromagnetic phase at $K / J \approx 1.233$, the latter, in its turn, again becomes of the second order at $K / J \approx 1.6$ and for large values of 
(a)

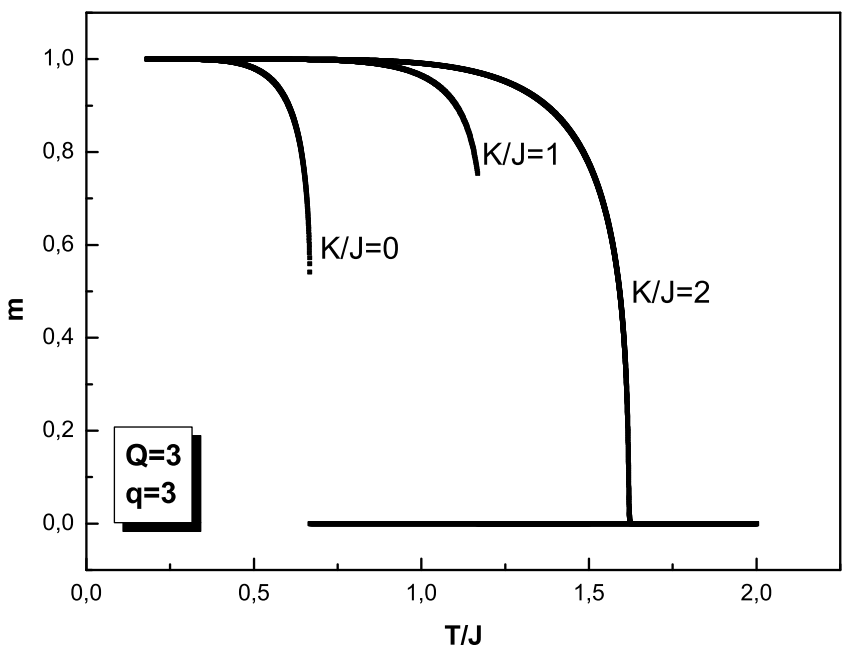

(b)

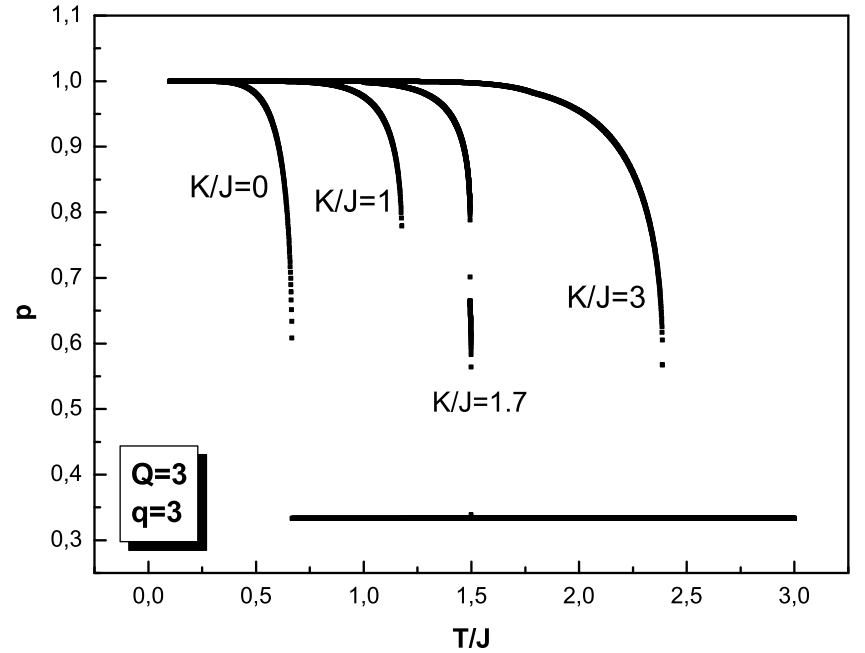

Figure 4: The temperature behavior of the order parameters for $Q=3$ and $q=3$. Here magnetization of the system undergoes a jump for small values of $K / J$ and becomes continuous beginning with $K / J \approx 1.84$. Another order parameter always remains discontinuous at the transition points. In the plots $p(T)$ for $K / J=1.7$ one can see that the transitions between "quadrupolar" and ferromagnetic phases is of the first order, whereas with the further increase of $K / J$ in becomes continuous.

$K / J$ it becomes parallel to the $K / J$ axis which means that the transition temperature between quadrupolar and ferromagnetic phases is unaffected by the value of $K$ beginning with $K / J \approx 4$.

The situation is quite different for $Q>2$. In Fig. 4 the plots of zero field magnetization and quadrupolar moment for $Q=3$ and $q=3$ are presented. In this case both $m$ and $p$ are discontinuous at the critical temperatures. Moreover, this feature is preserved for $p$ for all values of $K / J$, whereas $m$ becomes continuous after $K / J \approx 1.84$. the corresponding phase diagram is presented in Fig. 5. Here one can see a triple point at $K / J \approx 1.67$ where the merging of the three lines of the first order transitions takes place. There is also a tricritical point at $K / J \approx 1.84$ which separates the region of the first and the second order phase transitions on the line between quadrupolar and ferromagnetic phases. The line, as in case of $Q=2$, goes to plateau beginning with $K / J \approx 5$, so the transition temperature again does not depend on $K$ for $K \geq 5 J$. In general, for $Q>3$ the pase diagrams of the model under consideration have the same topology as in case of $Q=3$. The main feature is the decrease of the distance between triple and tricritical points with the increase of the $Q$. So, for $Q=30$ one can obtain that this distance is of $10^{-2}$ order with $K / J$. Apparently, they have never shrunk to a single point at finite values of $Q$, but it could be the case when $Q \rightarrow \infty$. The value of critical spin component number $Q_{c}$ at which the phase transitions become of the first order at $K=0$ in the 


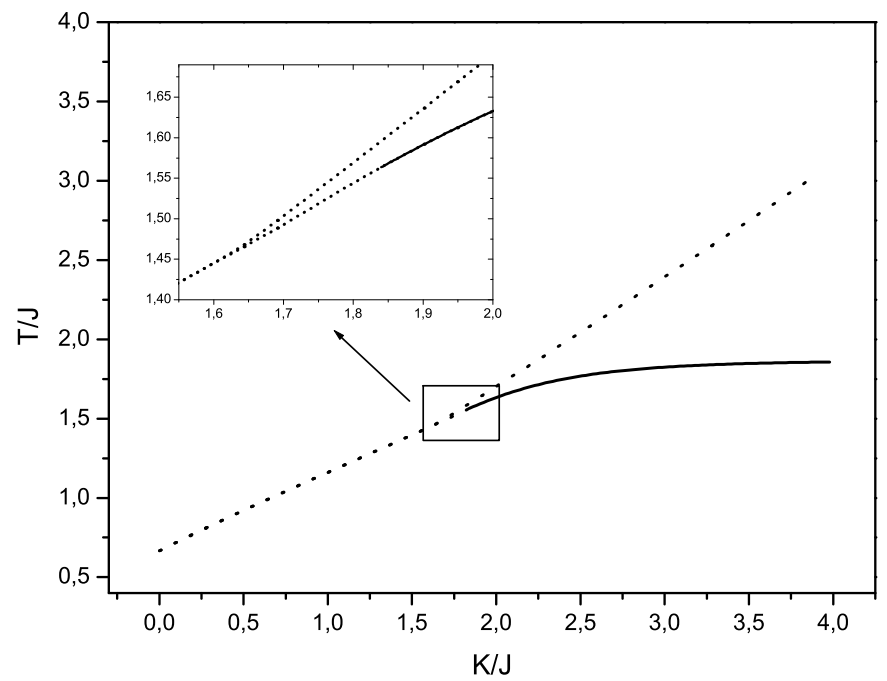

Figure 5: The phase diagram of the model for $Q=3$ and $q=3$. Solid line corresponds to the second order transitions, dotted - to the first order transitions. The inset shows the merge point of the three first order lines (triple point): disordered-ferromagnetic, disordered-"quadrupolar" and "quadrupolar"-ferromagnetic. In this point at $K / J \approx 1.67$ these three phase are in the equilibrium. The tricritical point on the line between "quadrupolar" and ferromagnetic phases is situated rather close to the triple point. The distance between them tends to zero with the increase of $Q$. 
Face-cubic model on Bethe lattice is exactly equal to 3, which coincides with the mean-field results at $q \rightarrow \infty$, but, in contrast to that, on Bethe lattice this value is unaffected by the value of coordination number $q$. Obviously, this is the direct consequence of the fact, that the Bethe-Pierels approximation become exact on the Bethe lattice.

\section{Summary}

We have considered very general classical spin model with cubic symmetry on the Bethe lattice. First of all, for the underlying Cayley tree, which is the planar graph, we have presented the partition function of the model as a double power series in terms of graph expansions for arbitrary planar graphs. This double graph expansion representing the known formula for linear chain was evaluated exactly in case of Cayley tree. The expression for free energy per spin in the thermodynamic limit in this case was found to be continuous in temperature. This is in full agreement with general statement about mechanisms of phase transitions on recursive lattices. In the thermodynamic limit the influence of the huge amount of boundary sites, which in contrast to all other sites have only one neighbor, precludes the system from undergoing any phase transitions in the sense usually understood in the thermodynamics of "normal" systems. Then, getting rid of all boundary sites by formal putting of the coordination number of all sites equal to $q$, thus, passing to the completely homogeneous connected recursive graph, the Bethe lattice, we have applied the methods of the dynamical systems theory or, more precisely, the theory of discrete mappings. In this technique one exploits the self-similarity of the Bethe lattice and establishes a connection between the thermodynamic quantities defined for the lattices with different number of sites. We have used the method in which this connection is given by the $\mathrm{RR}$ for some formal variables $x$ and $y$ which have no direct physical meaning but can be used to determine any thermodynamic function of the system. The key point of this approach is the iterative procedure for the system of RR $x_{n}=f_{1}\left(x_{n-1}, y_{n-1}\right), y_{n}=f_{2}\left(x_{n-1}, y_{n-1}\right)$, where $n$ can be regarded as the number of generations into the Bethe lattice. Thus, doing the iterations one approaches the thermodynamic limit $n \rightarrow \infty$. However, actually the iterative procedure is terminated for some finite number of iterations by the fixed point (which is the case for ferromagnetic couplings)or by s-cycles, which takes place in the antiferromagnetic case. There is also more complicated case of the chaotic behavior of the iterative sequence but it is out of the topic of the article. We have identified the different thermodynamic phases of the system in ferromagnetic case $(J>0, K>0)$ (disordered, partially ordered and completely ordered) with different types of the fixed points of RR. According to that we have obtained the phase diagrams of the model which are found to be different for $Q \leq 2$ and $Q>2$. The former case contains three tricritical points whereas the latter just one tricritical and one triple points. 


\section{Acknowledgments}

The authors are grateful to N. S. Izmailian for useful discussions. This work was partly supported by grants ISTC A-655 and ISTC A-820. L. N. Ananikyan thanks NFSAT (GRSP16/06) for partly support.

\section{References}

[1] A. Ahahrony and A. D. Bruce, Phys. Rev. Lett. 33, 427 (1974).

[2] A. D. Bruce and A. Aharony, Phys. Rev. B 11, 478 (1975).

[3] A. Aharony, in Phase Transition and Critical Phenomena, ed. by C. Domb and J. Lebowitz (Academic Pess, New York, 1976), vol. 6, p. 357.

[4] A. Pelissetto and E. Vicari, Phys. Rept. 368, 549 (2002).

[5] P. Calabrese and A. Celi, Phys. Rev. B 66, 184410 (2002).

[6] P. Calabrese, A. Pelissetto, and E. Vicari, Phys. Rev. B 67, 054505 (2003).

[7] P. Calabrese, E. V. Orlov, D. V. Pakhnin, and A. I. Sokolov, Phys. Rev. B 70, 094425 (2004).

[8] N. C. Bartelt, T. L. Einstein, and L. T. Wille, Phys. Rev. B 40, 10759 (1989); T. Aukrust, M. A. Novotny, P. A. Rikvold, and D. P. Landau, Phys. Rev. B 41, 8772 (1990).

[9] T. Chou and D. R. Nelson, Phys. Rev. E 48, 4611 (1993).

[10] S. Bekhechi, A. Benyoussef, and N. Moussa, Phys. Rev. B 61, 3362 (2000).

[11] H. Kleinert, S. Thoms, and V. Schulte-Frohlinde, Phys. Rev. B 56, 14428 (1997).

[12] B. N. Shalaev, S. A. Antonenko, and A. I. Sokolov, Phys. Lett. A 230, 105 (1997).

[13] D. V. Pakhnin and A. I. Sokolov, Phys. Rev. B 61, 15130 (2000).

[14] D. V. Pakhnin and A. I. Sokolov, Phys. Rev. B 64, 094407 (2001).

[15] P. Calabrese, A. Pelissetto and E. Vicary, Acta Phys. Slov. 52 , 311 (2002).

[16] Z. Usatenko and J. Spałek, J. Phys. A 37, 7113 (2004).

[17] H. Kachkachi and E. Bonet, Phys. Rev. B 73, 224402 (2006).

[18] D. Kim, P. M. Levy and L. F. Uffer, Phys. Rev. B 12, 989 (1975). 
[19] D. Kim and P. M. Levy, Phys. Rev. B 12, 5105 (1975).

[20] D. Kim, P. M. Levy, and J. J. Sudano, Phys. Rev. B 13, 2054 (1976).

[21] A. Aharony, J. Phys. A 10, 389 (1977).

[22] E. K. Riedel, Physica A 106, 110 (1981).

[23] B. Nienhuis, E. K. Riedel, and M. Schick, Phys. Rev. B 27, 5625 (1983).

[24] R. Baxter Exactly Solved Models in Statistical Mechanics (Academic Press, New York 1982).

[25] See, for example, H. G. Schuster Deterministic Chaos (Weinheim: Physik, 1984).

[26] T. P. Eggarter, Phys. Rev. Lett. 9, 2989 (1974).

[27] E. Müller-Hartmann and J. Zittartz, Phys. Rev. Lett. 33, 893 (1974); Z. Phys. B 22, 59 (1975); E. Müller-Hartmann, Z. Phys. B 27, 161 (1977).

[28] C. J. Thompson, J. Stat. Phys. 27, 441 (1982).

[29] T. Stošić, B. D. Stošić and I. P. Fittipaldi, Physica A 320, 443 (2003); Physica A 355, 346 (2005).

[30] M.-S. Chen, L. Onsager, J. Bonner, and J. Nagle, J. Chem. Phys. 60, 405 (1974).

[31] J. L. Monroe, J. Stat. Phys. 65, 255 (1991); J. Stat. Phys. 67, 1185 (1992).

[32] N. S. Ananikian, S. K. Dallakian, N. Sh. Izmailian and K. A. Oganessyan, Fractals, 5, 175 (1997).

[33] T. A. Arakelyan, V. R. Ohanyan, L. N. Ananikyan, N. S. Ananikian and M. Roger, Phys. Rev. B 67, 024424 (2003).

[34] P. D. Gujrati, Phys. Rev. Lett. 74, 809 (1995).

[35] J. Vannimenus, Z. Phys. B 43, 141 (1981).

[36] C. S. O. Yokoi, M. J. de Oliveira, S. R. Salinas, Phys. Rev. Lett. 54, 163 (1985); M. H. R. Tragtenberg, C. S. O. Yokoi, Phys. Rev. E 52, 2187 (1995).

[37] A. Z. Akheyan and N. S. Ananikian, J. Phys. A 25, 3111 (1992); N. S. Ananikian, R. R. Scherbakov, J. Phys. A 27, L887 (1994); N. S. Ananikian, S. K. Dallakian, B. Hu, N. Sh. Izmailian and K. A. Oganessyan, Phys. Lett. A 248, 381 (1998).

[38] P. D. Gujrati, Phys. Rev. Lett. 53, 2453 (1984); J. Chem. Phys. 98, 1613 (1993). 
[39] Vl. V. Papoyan and R. R. Scherbakov, J. Phys. A 28, 6099 (1995); Fractals 4, 105 (1996).

[40] J. C. Wheeler and B. Widom, J. Chem. Phys. 52, 5334 (1970).

[41] A. Z. Akheyan and N. S. Ananikian, Phys. Lett. A 186, 171 (1994); JETP 107, 196 (1995).

[42] A. Erdiç, O. Canko and A. Albayrak, J. Magn. Magn. Mater. 303, 185 (2006).

[43] J. L. Monroe, J. Phys. A 29, 5421 (1996).

[44] M. Eckstein, M. Kollar, K. Byczuk, and D. Vollhardt, Phys. Rev. B 71, 235119 (2005).

[45] N. S. Ananikian, S. K. Dallakian, B. Hu, Complex Systems 11, 213 (1997).

[46] N.S. Ananikian, A.R. Avakian and N.S. Izmailian, Physica A 172, 391 (1991); A. Z. Akheyan, and N. S. Ananikian, J. Phys. A 29, 721 (1996).

[47] E. Albayrak and M. Keskin, Eur. Phys. J. B 24, 505 (2001); C. F. Delale, Int. J. Mod. Phys. 33, 1523 (1998).

[48] C. Ekiz, J. Magn. Magn. Mater. 293, 759 (2005); 293, 913 (2005); Physica A 347, 353 (2005); A 353, 286 (2005).

[49] A. E. Alahverdian, N. S. Ananikian and S. K. Dallakian, Phys. Rev. E 57, 2452 (1998); N. S. Ananikian, R. G. Ghulghazaryan, Phys. Lett. A 277, 249 (2000); R. G. Ghulghazaryan, N. S. Ananikian and P. M. A. Sloot, Phys. Rev. E 66, 046110 (2002); R. G. Ghulghazaryan, N.S. Ananikian, J.Phys. A 36, 6297 (2003).

[50] F. Y. Wu, Rev. Mod. Phys. 54, 235 (1982).

[51] B. Balobas, Modern Graph Theory (Springer, New York, 1998). 